\title{
Comparative transcriptome analysis reveals regulatory genes involved in cold tolerance and hypoxic adaptation of high-altitude Tibetan bumblebees
}

\author{
Yanjie Liu ${ }^{1}$, Hongyan $\mathrm{JIN}^{2}$, Muhammad NAEEM ${ }^{1,3}$, Jiandong AN ${ }^{1}$ \\ ${ }^{1}$ Key Laboratory for Insect-Pollinator Biology of the Ministry of Agriculture and Rural Affairs, Chinese Academy of \\ Agricultural Sciences, Institute of Apicultural Research, No 1 Beigou, Xiangshan, Haidian, Beijing 100093, China \\ ${ }^{2}$ Tibet Vocational Technical College, Lhasa 850030, China \\ ${ }^{3}$ Department of Zoology, Wildlife \& Fisheries, PARS Campus, University of Agriculture, Faisalabad 38000, Pakistan
}

Received 20 February 2020 - Revised 8 June 2020 - Accepted 21 July 2020

\begin{abstract}
China has many habitats supporting a wide diversity of bumblebees, some species of which are limited in distribution to the Tibet Plateau. The high-altitude Tibet Plateau, which has low air density as well as extremely low temperatures, strains the energy requirements for flight in most insects. However, high-altitude bumblebees have adapted to the harsh conditions of this type of habitat. Bumblebees are a particular group of insect pollinators that exclusively utilize carbohydrates from flowers for energy to sustain flight. Here, gene expression was compared between low-altitude species and Bombus longipennis, and the genes upregulated in B. longipennis were involved in aerobic metabolism, such as the oxidative phosphorylation and citric acid cycle (TCA cycle) pathways. Furthermore, a conjoint analysis of the transcriptomes of six bumblebee species from the high-altitude Tibet Plateau and two bumblebee species from the low-altitude North China Plain showed that 19 genes were commonly upregulated in high-altitude species. Among these 19 commonly upregulated genes, Pfkl was enriched in multiple glycometabolic pathways, which are the main energy pathways in bees; this upregulation enhanced the aerobic and anaerobic glycolysis processes to produce more ATP molecules to supply energy for high-altitude bumblebee flight under severe cold conditions. In addition, glycolysis was enhanced by two other genes, Racl and AAC2. Relative quantitative real-time PCR was used to verify that the three genes Pfk1, Racl, and $A A C 2$ were upregulated in the six main bumblebee species inhabiting the Tibet Plateau.
\end{abstract}

pollinators / bumblebees / Tibet Plateau / transcriptome / energy metabolism / pfk1

\section{INTRODUCTION}

High-altitude environments have significantly lower air density and oxygen content than lowaltitude environments and sustain low temperatures (Altshuler and Dudley 2006; Dillon et al.

Electronic supplementary material The online version of this article (https://doi.org/10.1007/s13592-020-00795-w) contains supplementary material, which is available to authorized users.

Corresponding author: J. An, anjiandong@ caas.cn Manuscript Editor: Klaus Hartfelder
2006). Altitudinal reductions in oxygen content challenge insect flight, and low temperatures may lead to flight deficiency for many insect groups at high elevations (Hodkinson 2005). However, some insects, such as bumblebees, which are effective pollinators, are capable of adapting to these environments and maintaining normal flight at high altitudes (Mani 1968). Bumblebees have been found at altitudes over $5000 \mathrm{~m}$ in Alpine Mountain and are capable of flight at simulated altitudes above $8000 \mathrm{~m}$ (Williams et al. 2010; Dillon and Dudley 2014). Moreover, some bumblebee species inhabit only elevations greater than 
$3500 \mathrm{~m}$ on the Tibet Plateau (Royden et al. 2008; An et al. 2014). Remarkably, low air density and low temperature still limit flight even in insects that possess a powerful respiratory system by more mechanisms than hypoxia (Wigglesworth 1972; Harrison and Roberts 2000; Ghabrial et al. 2003; Dillon and Frazier 2006; Dillon et al. 2006). However, in contrast to the respiratory systems of insects, humans, mammals, and birds have evolved the ability to adapt to high-altitude hypoxia, and the genetic basis of this adaptation has been revealed. High-altitude adaptation among Tibetan people includes positive selection on the genes EGLN1 and PPARA, which are involved in hypoxia-response pathways, according to genome and exome data (Beall et al. 2010; Bigham et al. 2010; Simonson et al. 2010; Yi et al. 2010; Peng et al. 2011; $\mathrm{Xu}$ et al. 2011). Another gene, EPAS1, which encodes hypoxia-inducible factor $2 \alpha$, is downregulated as part of the molecular basis of adaptation to high-altitude hypoxia in Tibetan people (Peng et al. 2017). Based on genome analysis, other mammals, including Tibetan yak, antelopes, Tibetan dogs, Tibetan horses, and gray wolves, share a mechanism similar to that of humans in adaptation to high-altitude environments (Qiu et al. 2012; Ge et al. 2013; Gou et al. 2014; Zhang et al. 2014; Liu et al. 2019). In addition to mammals, birds from the Tibet Plateau evolved increased hemoglobin $(\mathrm{Hb})-\mathrm{O}_{2}$ affinities to adapt to high-altitude environments, allowing normal flight (Projecto-Garcia et al. 2013; Galen et al. 2015; Natarajan et al. 2015; Natarajan et al. 2016; Zhu et al. 2018). Notably, experimentally evolved Drosophila melanogaster populations were shown to share some hypoxia-tolerancerelated genes with high-altitude human populations (Miyazawa et al. 2017). However, the physiological and metabolic mechanisms of adaptation to cold temperatures in high-altitude habitats for bees and other insects remain unknown.

Flying insects exhibit higher oxygen consumption than runners when sustaining high metabolic rates in flight muscle (Harrison and Roberts 2000). These high metabolic glycolytic flux demands are essential for flying insects, as this metabolic pathway is the sole route providing flight fuel (Sacktor and Wormser-Shavit 1966; Sacktor 1976; Suarez 2000). Low temperature reduces metabolic rates and changes the dynamics of flight muscle, limiting flight in high-altitude insects (Josephson 1981; Huey and Kingsolver 1989; Scaraffia and Gerez de Burgos 2000). Moreover, bees, including honeybees and bumblebees, exclusively utilize carbohydrates to power their flight; thus, these insects support flight by using sugars from plant nectar rather than using the fat body (Suarez et al. 2005). Nectar is composed mainly of carbohydrates and water, and its main constituents include sucrose, fructose, and glucose (Roy et al. 2017). Many studies have reported that bee flight muscles utilize hexose sugars to provide energy for flight (Gmeinbauer and Crailsheim 1993; Suarez 2000; Blatt and Roces 2001). Apart from the anaplerotic role played by proline, carbohydrate oxidation predominates over fatty acid oxidation as the major source of energy for flight in orchid bees (Suarez et al. 2005). As the only energy-producing pathway of carbohydrate metabolism, glycolysis generally includes aerobic and anaerobic glycolytic flux. Under normal oxygen conditions, cells metabolize glucose to $\mathrm{CO}_{2}$ and $\mathrm{H}_{2} \mathrm{O}$ in an oxygen-dependent and highly energy-efficient manner, mainly relying on the tricarboxylic acid cycle and oxidative phosphorylation in the mitochondria (Gatenby and Gillies 2004). Cells switch from this aerobic pathway to an anaerobic glycolytic pathway for ATP production under hypoxia (Brahimi-Horn et al. 2007).

Based on an investigation of bumblebee species for approximately 10 years in China, we found that several species were distributed only at high elevations on the Tibet Plateau, while other species inhabited low elevations in North China (Williams et al. 2016; Huang and An 2018). The high-elevation bumblebee species have evolved and adapted to the Tibet Plateau environment, including cold temperatures and hypoxic conditions, but the genetic basis of this adaptation is currently unknown. To explore the genetic basis of high-altitude adaptation, we sequenced and compared the transcriptomes of six high-altitude bumblebee species from the Tibet Plateau and two low-altitude bumblebee species from North China to identify common regulatory factors contributing to high-altitude adaptation in bumblebees. 


\section{MATERIALS AND METHODS}

\subsection{Bumblebee samples and transcriptome sequencing}

Six representative high-altitude bumblebee species, including Bombus longipennis (E $92.29164^{\circ}, \mathrm{N} 30.08586^{\circ}$, elevation $\left.4337 \mathrm{~m}\right), B$. difficillimus (E 82.25945 ${ }^{\circ}$ N $30.61903^{\circ}$, elevation $4844 \mathrm{~m}$ ), B. rufofasciatus (E 85.47504, $\mathrm{N}$ $28.83427^{\circ}$, elevation $4746 \mathrm{~m}$ ), B. semenovi (E $92.90090^{\circ}, \mathrm{N} 34.75130^{\circ}$, elevation $4726 \mathrm{~m}$ ), B. superbus (E 91.98893, N 32.98648, elevation $4891 \mathrm{~m}$ ), and B. waltoni (E 91.67894, $\mathrm{N}$ $32.19394^{\circ}$, elevation $4651 \mathrm{~m}$ ), from the Tibet Plateau, and two representative low-altitude species, B. ignitus (E 117.23738 ${ }^{\circ}$, N 35.82117º, elevation $245 \mathrm{~m}$ ) and $B$. patagiatus (E $131.0818^{\circ}, \mathrm{N} 44.14578^{\circ}$, elevation $272 \mathrm{~m}$ ), from the North China Plain, were used in this study (Figure 1). Bumblebee samples were collected in July-August 2017-2018. Three worker bees of each bumblebee species were captured, the abdomen was removed, and the head and thorax of each bee were frozen in liquid nitrogen to be used for RNA extraction, thus generating three replicates per species. RNA was extracted by TRIzol Reagent (Life Technologies, USA) and dissolved in RNase-free water. The purity, concentration, and integrity of the extracted RNA samples were assessed by a NanoDrop 2100 (Thermo Scientific, USA), Qubit 2.0 (Qubit), and an Agilent 2100 (Agilent, USA) instrument, respectively. mRNA was enriched using oligo (dT) magnetic beads and random fragmentation. The fragmented mRNA was used for synthesis of the first cDNA strand by reverse transcription, and second-strand cDNA synthesis was performed using DNA polymerase I and RNase H. Then, the ends of the doublestranded cDNA were repaired, a single "A" base was added to the ends, and adapters were ligated; finally, the samples were purified with AMPure XP beads to select fragments of 150-200 bp, which were used for PCR amplification. A cDNA library for each sample was constructed, the PCR products were sequenced on the Illumina HiSeq 4000 platform (Novogene Co., Ltd., Beijing, China), and paired-end reads were generated.

\subsection{Data processing, genome alignment, and novel gene prediction}

The raw image data files from the Illumina HiSeq 4000 were transformed into the original sequenced reads (raw reads) by CASAVA 1.8 base calling analysis and processed with inhouse Perl scripts. Clean data (clean reads) were obtained by eliminating the low-quality reads (reads containing an adapter, reads containing poly-N, and reads with Qphred $\leq 20$, equivalent to reads with base call accuracy less than 99\%) from raw reads. Clean data with high quality were aligned to the reference genome of Bombus terrestris (Sadd et al. 2015). A mapping analysis was performed by HISAT software with the default parameters (Sacktor 1976). Then, novel genes were predicted by StringTie software and annotated using the Pfam database.

\subsection{Differentially expressed gene analysis and KEGG enrichment}

Calculating the expected number of fragments per kilobase of transcript sequence per million base pairs sequenced (FPKM) is the most commonly used method of estimating gene expression levels (Sharma 2011). The read count for each gene was obtained by mapping the clean reads back to the reference genome. Genes with FPKM $>1$ were considered to be expressed. Differential expression of genes was analyzed and calculated based on the read count value using DESeq2 (Nunes et al. 2016). Based on the negative binomial distribution model, DESeq provides statistical routines to determine differential expression. The $p$ values were adjusted using Benjamini and Hochberg's approach for controlling the false discovery rate (Xie et al. 2011). The thresholds for judging significant differences in gene expression levels between any two groups were padj $<0.05$ and $\log 2$ (fold change) $\geq 1$ or $\log 2$ (fold change) $\leq-1$. The $\mathrm{p}$-adjusted (padj) was the corrected $p$ value, and a small padj value of DEGs indicated that the differential expression was highly significant (Sacktor and WormserShavit 1966). The KEGG enrichment of 


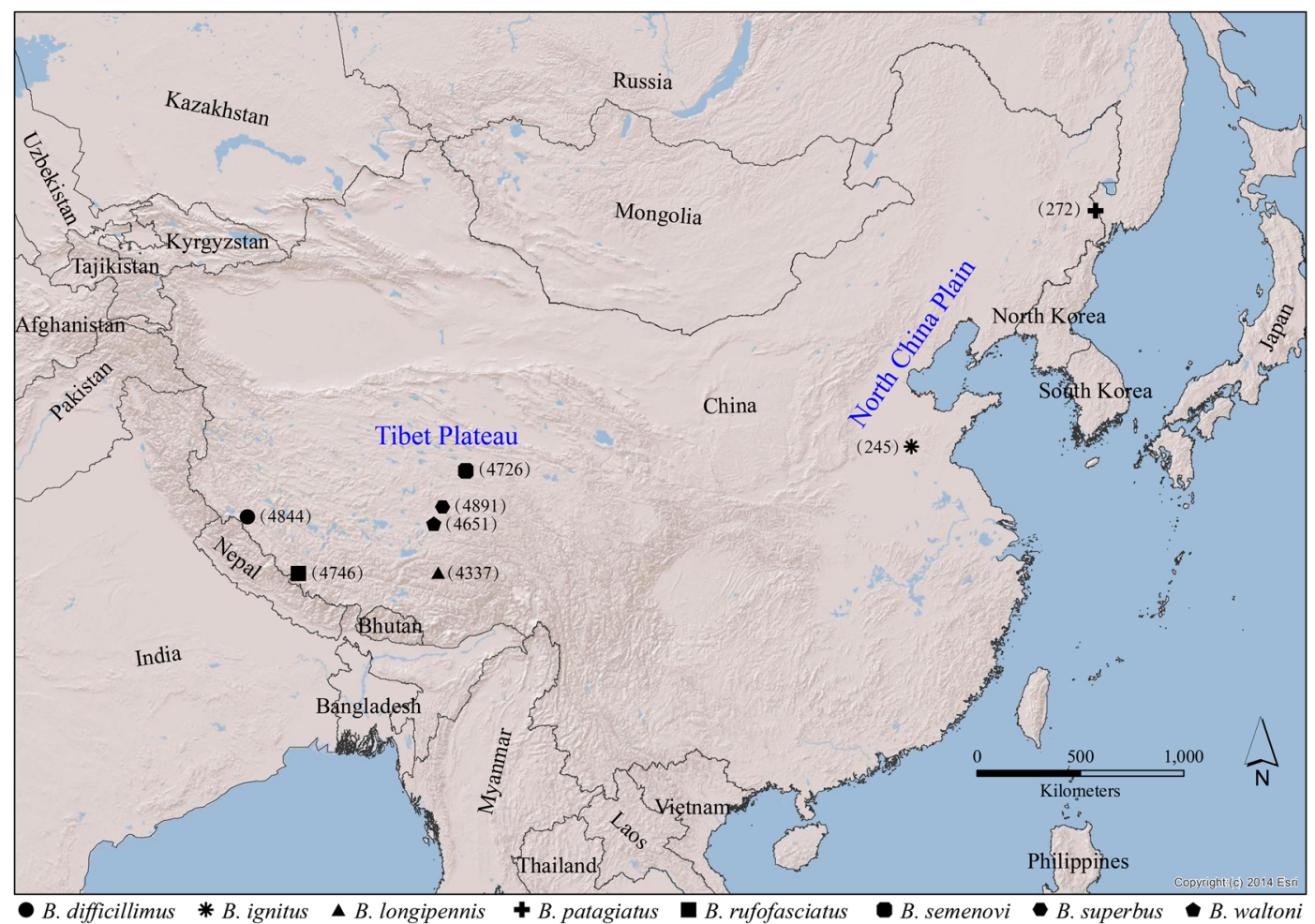

Figure 1. Relief map of mainland China showing the sampling sites and their elevations (m) of the eight bumblebee species in this study. Six species from the high-altitude Tibet Plateau, including B. longipennis, B. waltoni, B. rufofasciatus, B. difficillimus, B. superbus, and B. semenovi, and two species from the low-altitude North China Plain, including B. patagiatus and B. ignitus, were collected. The international boundaries are shown in gray with the names of countries in gray. The map was created with ArcGIS v 10.0.

differentially expressed genes was performed by the KOBAS 3.0 server (http://http://kobas.cbi. pku.edu.cn/) with a $p$ value $<0.05$ (Xie et al. 2011).

\subsection{Relative quantitative real-time $P C R$ verification}

Total RNA extraction and RT-PCR were performed as previously described for preparation for RNA-Seq. All relative quantitative real-time PCR (qRT-PCR) samples were run on an ABI 7500 system (Applied Biosystems, Thermo Fisher Scientific, America) with Bestar® Sybr Green qPCR Mastermix enzyme. Each reaction was performed with a total volume of $20 \mu \mathrm{L}$ that contained $5 \mu \mathrm{L}$ first-strand cDNA as a template and processed with a preincubation program of $5 \mathrm{~min}$ at $95^{\circ} \mathrm{C}$, followed by 40 cycles of $15 \mathrm{~s}$ at $95{ }^{\circ} \mathrm{C}, 45 \mathrm{~s}$ at $60^{\circ} \mathrm{C}$, and $10 \mathrm{~min}$ at $72^{\circ} \mathrm{C}$. Gene-specific primers were designed with Primer Premier 5 (Table S1). The bumblebee $\alpha$-actin gene and arginine kinase gene were used as internal references for gene expression. Gene expression levels were calculated using the $2^{-\Delta \Delta C t}$ method (Mor et al. 2011). The mean threshold cycle values for each gene were obtained from three independent PCRs of different individuals.

\subsection{AA alignment and modeled structure analysis}

The AA alignment of pfk1, rac1, and AAC2 proteins was performed using the Clustal Omega server (https://www.ebi.ac.uk/Tools/msa/clustalo/) and the ESPript3 server (http://espript.ibcp. 
fr/ESPript/ESPript/). The structures were modeled by the SWISS-MODEL server (https://www. swissmodel.expasy.org/) and analyzed by PyMOL (DeLano Scientific LLC).

\section{RESULTS}

\subsection{Transcriptome assembly and gene annotation}

The head and thorax transcriptomes of six high-altitude and two low-altitude bumblebee species were assembled based on the genome of $B$. terrestris. The $\mathrm{Q} 30$ values of clean reads of all sequenced samples were above $90 \%$, and the GC contents of all sequenced samples were approximately $40 \%$ (Table S3). The mapping ratios of the sequenced samples to the reference genome are shown in Table I. The novel genes of all the bumblebee species were predicted by StringTie software and annotated in the Pfam database. Differentially expressed genes were identified between each high-altitude bumblebee species and each of the low-altitude species, B. ignitus and B. patagiatus (Figure 2).

\section{2. $B$. longipennis belongs to the same subgenus as $B$. ignitus and $B$. patagiatus and shows upregulation of genes related to aerobic metabolism}

Similar to B. ignitus and B. patagiatus, the high-altitude bumblebee $B$. longipennis belongs to the subgenus Bombus s. str. Compared to $B$. ignitus and B. patagiatus, B. longipennis had a total of 1029 differentially expressed genes (Figure S1). These differentially expressed genes consisted of 630 upregulated genes and 339 downregulated genes (Figure 3a). The upregulated genes were mainly involved in the metabolic pathways, oxidative phosphorylation, glycolysis/ gluconeogenesis, carbon metabolic pathways, and TCA cycle pathways (Figure 3b) and were specifically involved in the energy metabolism of aerobic respiration. However, the downregulated genes were mostly involved in ribosome pathways, longevity-regulating pathways (multiple species), xenobiotic metabolism by cytochrome
P450, and drug metabolic (cytochrome P450) pathway (Figure 3c).

\subsection{The six high-altitude bumblebee species shared 19 commonly upregulated genes and 141 commonly downregulated genes}

Moreover, compared to B. ignitus, the six high-altitude species shared 32 commonly upregulated genes (Figure 4a) and 389 commonly downregulated genes (Figure 4b). In addition, compared with $B$. patagiatus, the highaltitude bumblebees had 83 upregulated genes and 642 downregulated genes in common (Figure $4 \mathrm{c}$ and $d$ ). Further, Venn analysis of these common differentially expressed genes showed that only 19 commonly upregulated genes, including three novel genes, were shared by all six high-altitude species compared to both low-altitude species (Figure 4e and Table S3). There were 141 commonly downregulated genes, including 91 novel genes, found in the six high-altitude bumblebee species in comparison to the two lowaltitude species (Figure $4 \mathrm{f}$ and Table S3). The commonly downregulated genes were significantly involved in pentose and glucuronate interconversion, metabolism of xenobiotics by cytochrome P450, drug metabolic (cytochrome $\mathrm{P} 450$ ), FoxO signaling, glutathione metabolic, and longevity-regulating (multiple species) pathways (Figure 5a). For the 19 commonly upregulated genes, no significantly enriched pathways were found. However, the $P f k 1$ gene (LOC100648793, ATP-dependent 6-phosphofructokinase (PFK)) is involved in multiple metabolic pathways, especially glycometabolic pathways, such as the starch and sucrose metabolic pathways, pentose phosphate pathway, microbial metabolism in diverse environments, metabolic pathways, glycolysis/gluconeogenesis, amino sugar and nucleotide sugar metabolic pathways, carbon metabolic pathways, biosynthesis of amino acids, and galactose metabolism, fructose, and mannose metabolic pathways. Notably, $P f k 1$ was identified among the 19 commonly upregulated genes based on the 
Table I. Mapping ratio of the sequenced bumblebee samples to reference genome of Bombus terriestris

\begin{tabular}{|c|c|c|c|}
\hline Samples & Mapping ratio & Samples & Mapping ratio \\
\hline B. patagiatus 1 & $82.36 \%$ & B. rufofasciatus 1 & $53.16 \%$ \\
\hline B. patagiatus 3 & $84.2 \%$ & B. rufofasciatus 2 & $48.98 \%$ \\
\hline B. patagiatus 2 & $83.45 \%$ & B. rufofasciatus 3 & $53.54 \%$ \\
\hline B. ignitus 1 & $73.92 \%$ & B. semenovi 1 & $50.74 \%$ \\
\hline B. ignitus 2 & $78.37 \%$ & B. semenovi 2 & $51.42 \%$ \\
\hline B. ignitus 3 & $78.03 \%$ & B. semenovi 3 & $49.44 \%$ \\
\hline B. longipennis 1 & $74.66 \%$ & B. waltoni 1 & $23.96 \%$ \\
\hline B. longipennis 2 & $76.86 \%$ & B. waltoni 2 & $24.07 \%$ \\
\hline B. longipennis 3 & $77.61 \%$ & B.waltoni 3 & $24.83 \%$ \\
\hline B.difficillimus 1 & $52.21 \%$ & B. superbus 1 & $24.38 \%$ \\
\hline B. difficillimus 2 & $57.34 \%$ & B. superbus 2 & $24.37 \%$ \\
\hline B. difficillimus 3 & $53.76 \%$ & B. superbus 3 & $23.45 \%$ \\
\hline
\end{tabular}

database of the honeybee Apis mellifera (Figure 5b).

\subsection{Enhanced glycolysis is an efficient source of energy for high-altitude bumblebees}

In addition to $p f k 1, r a c 1$ and $A A C 2$, which are also part of the glycolysis pathway, were upregulated in the high-altitude bumblebees. Furthermore, the bumblebee Pfk protein shares similar binding pockets for ATP/ADP/ACP, fructose 6phosphate (F6P), and fructose 1,6-bisphosphatase (F6BP) to that of humans, and these pockets consist of the following residues: $\mathrm{G}^{127}, \mathrm{D}^{128}, \mathrm{G}^{129}$, and $\mathrm{S}^{130} ; \mathrm{R}^{102}, \mathrm{D}^{175}, \mathrm{R}^{217}, \mathrm{G}^{34}, \mathrm{E}^{273}, \mathrm{R}^{97}, \mathrm{R}^{210}$, and $\mathrm{R}^{301}$; and $\mathrm{H}^{307}, \mathrm{R}^{744}, \mathrm{R}^{481}, \mathrm{~N}^{542}, \mathrm{~T}^{538}, \mathrm{G}^{585}$, $\mathrm{S}^{540}, \mathrm{G}^{584}, \mathrm{E}^{639}, \mathrm{M}^{583}, \mathrm{H}^{671}, \mathrm{Q}^{674}, \mathrm{R}^{665}$, and $\mathrm{R}^{576}$ (Figure S2A and S2B). The residues located in the binding pocket were highly conserved between bumblebee Pfk1 and human Pfk1 (Figure S2C). Therefore, the function of Pfk1 might be conserved between bumblebees and humans, where it is involved in the first step of glycolysis commitment. Therefore, we suppose that after the bumblebee collects nectar from flowers, the major monosaccharides, fructose and glucose, of the nectar and pollen are transported into the cytoplasm by the plasma membrane glucose transporter (GLU) (Thaís et al. 2010; Archana and Archana
2016; Sadd et al. 2015); in the cytoplasm, Dglucose and $\mathrm{D}$-fructose are metabolized into $\beta$ $\mathrm{D}$-fructose-1, $6 \mathrm{P}_{2}$ by Pfk1, and then, $\beta$-D-fructose$1,6 \mathrm{P}_{2}$ is metabolized into pyruvate and produces the ATP that is hydrolyzed into ADP to provide energy (Figure 6). Under aerobic conditions, pyruvate enters the tricarboxylic acid cycle to produce ATP; in contrast, under hypoxic conditions, pyruvate is metabolized into lactate, which undergoes glycolysis again (Figure 6) (Berg et al. 2002). Most importantly, as the "gatekeeper" of glycolysis, the upregulated $P f k 1$ gene directly accelerates glycolysis, producing more ATP to protect high-altitude Tibetan bumblebees against cold temperatures. For high-altitude bumblebees, upregulation of the Racl gene might promote glucose uptake through GLUT4 in the flight muscles (Figure 6), as the racl gene can promote glucose uptake through GLU4 in skeletal muscles (Klip et al. 2014). Thus, the upregulation of the rac1 (LOC100650134) gene in bumblebees could promote the transport of glucose and fructose and facilitate glycolysis. The upregulation of the AAC2 (LOC100652161, ATP/ADP carrier protein) gene in the mitochondrial membrane facilitates the exchange of ATP and ADP by transporting ADP into mitochondria and ATP into the cytoplasm, indirectly stimulating glycolysis and the TCA cycle (Figure 6). Moreover, realtime PCR verification with a $p$ value $<0.05$ 


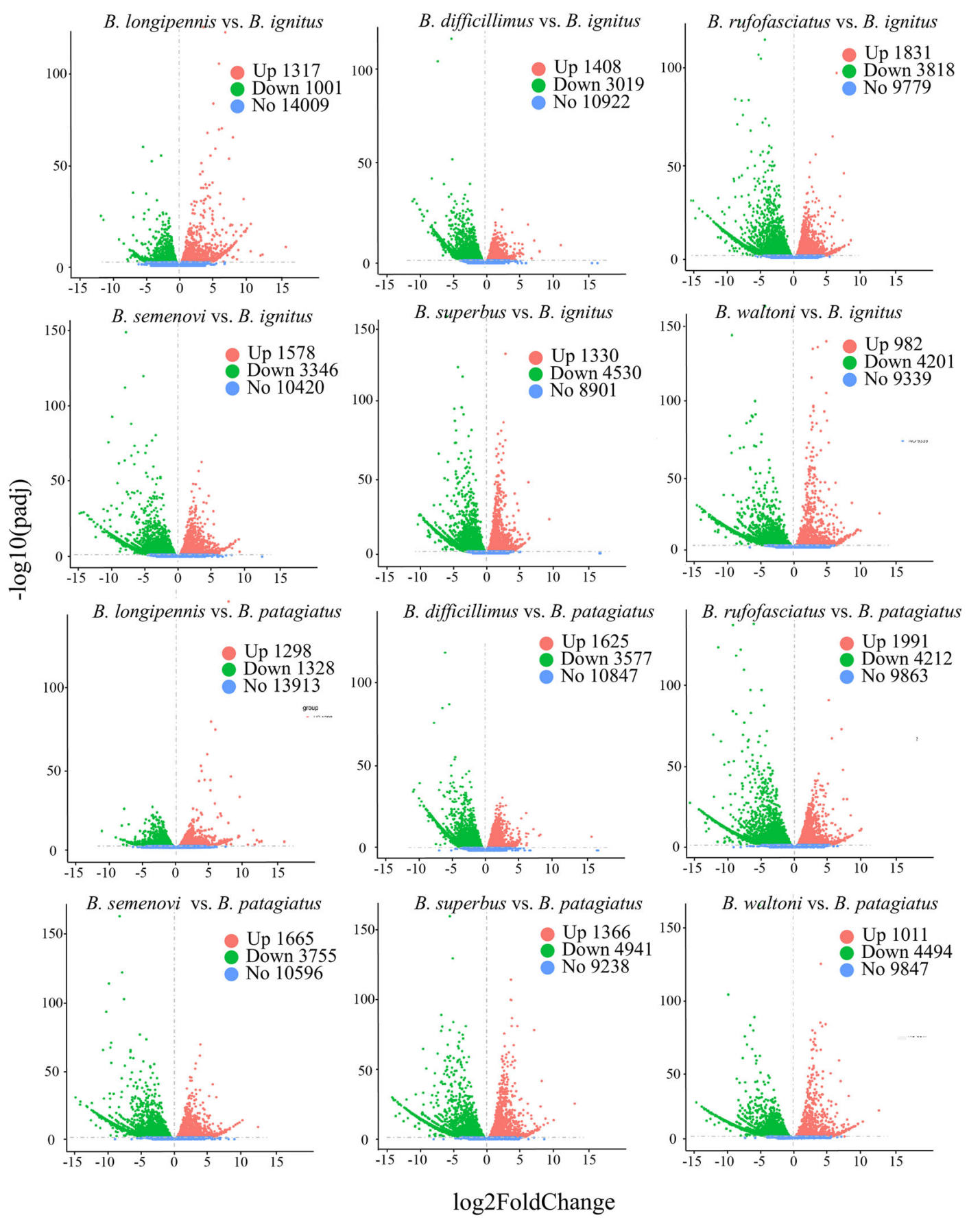

Figure 2. Volcano plot of differentially expressed genes between the six high-altitude bumblebee species in comparison to the two low-altitude species. Up represents upregulated genes and is shown by red dots, Down represents downregulated genes and is shown by green dots, and No represents no change in gene expression and is shown by blue dots.

showed that the Rac1, Pfk1, and AAC2 genes were upregulated in all sequenced high-altitude bumblebees (Figure 7). Importantly, the Pfkl and $A A C 2$ genes were strongly upregulated in 
a

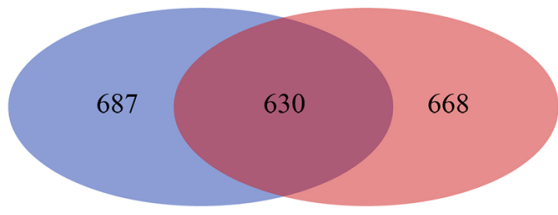

B. longipennis vs $B$. ignitus

B. longipennis vs B. patagiatus

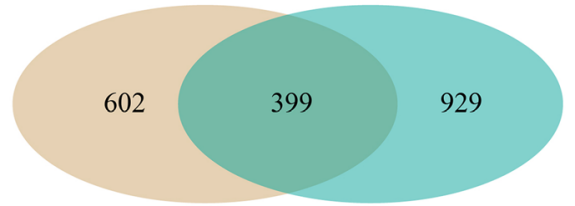

B. longipennis vs $B$. ignitus

B. longipennis vs B. patagiatus

b
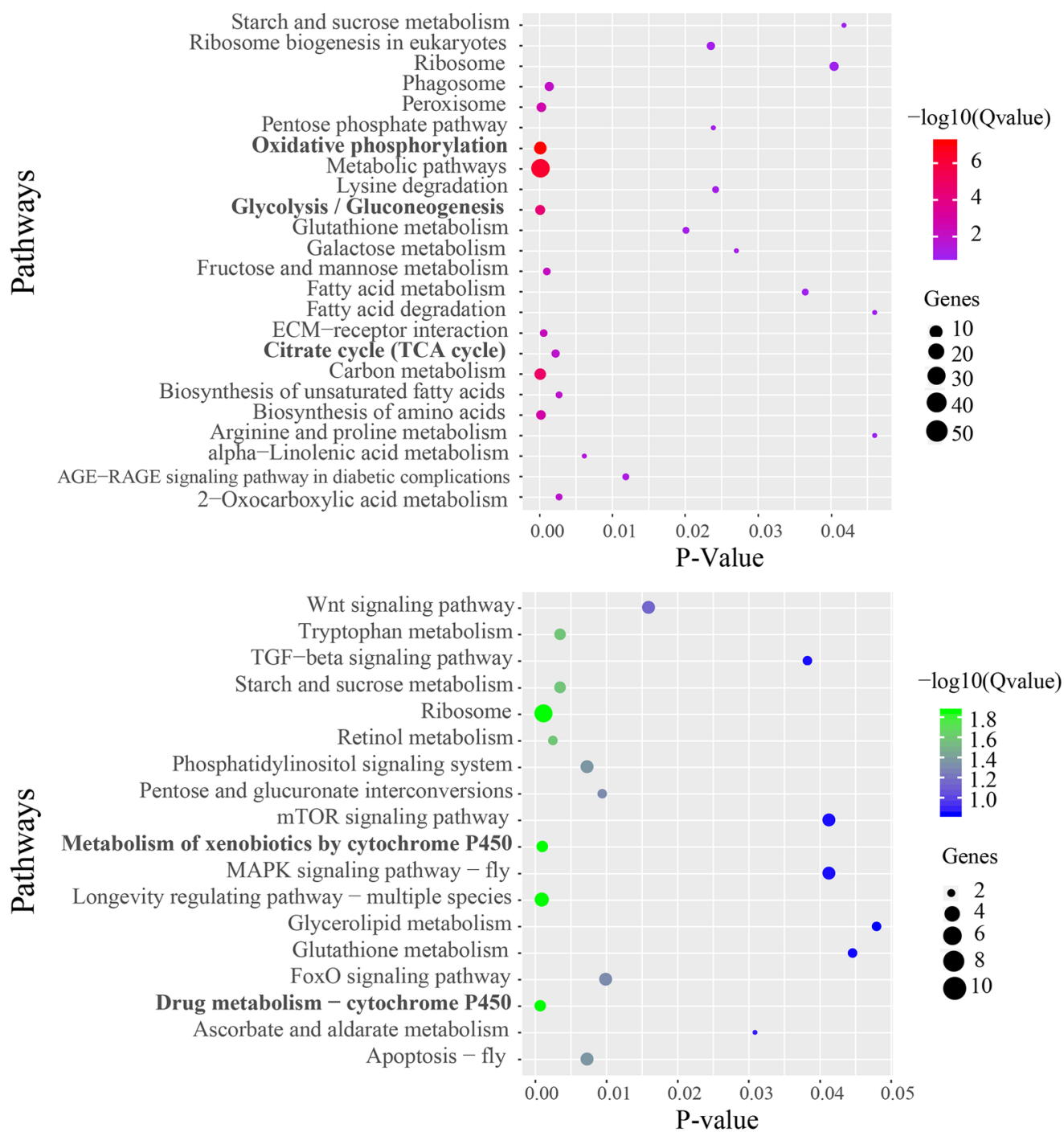

Figure 3. Venn diagram analysis and KEGG enrichment of the differentially expressed genes of $B$. longipennis in comparison to the two low-altitude bumblebee species. a Venn diagram of common differentially expressed genes in B. longipennis based on B. ignitus and B. patagiatus. b KEGG enrichment analysis of the upregulated genes in $B$. longipennis based on B. ignitus and B. patagiatus using the honeybee Apis mellifera database. c KEGG enrichment analysis of the downregulated genes in B. longipennis based on B. ignitus and B. patagiatus using the honeybee Apis mellifera database. 


\section{a}

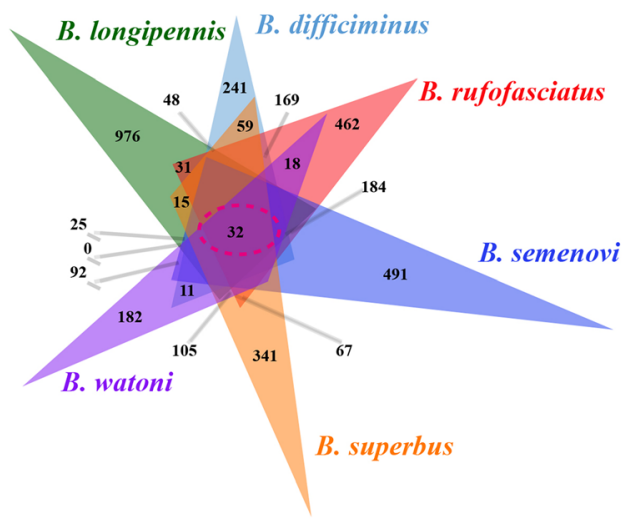

C

\section{B. longipennis B. difficiminus}

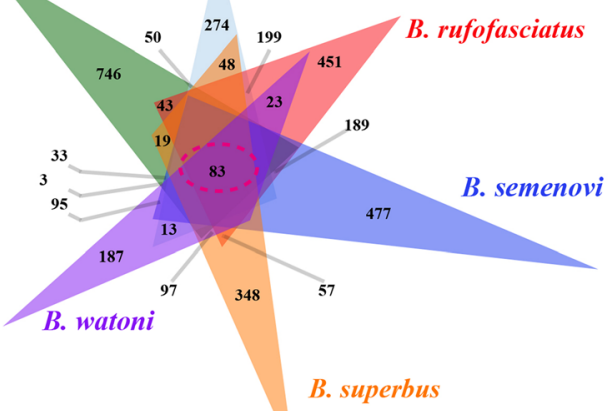

e

B. ignitus vs. B. patagiatus

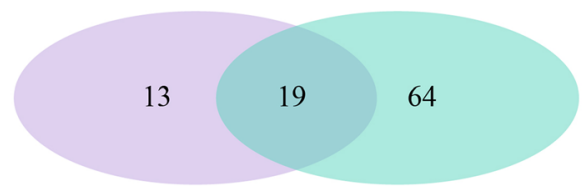

b

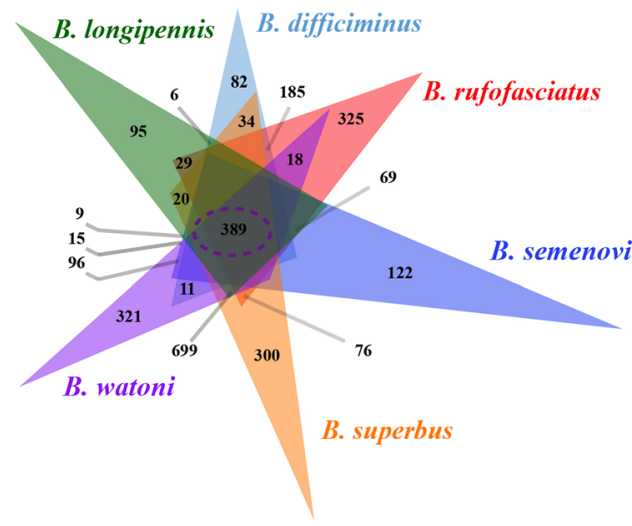

d

\section{B. longipennis B. difficiminus}

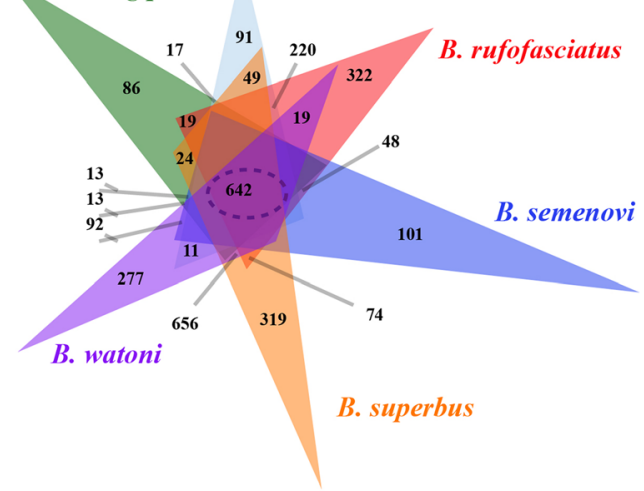

f

Figure 4. Venn diagram analysis of the differentially expressed genes between the six high-altitude bumblebee species and the two low-altitude bumblebee species. a Commonly upregulated genes in the six high-altitude bumblebee species compared to those in $B$. ignitus. b Commonly upregulated genes in the six high-altitude bumblebee species compared to those in $B$. patagiatus . c Commonly downregulated genes in the six highaltitude bumblebee species compared to those in B. ignitus . d Commonly downregulated genes in the six highaltitude bumblebee species compared to those in B. patagiatus. e Commonly upregulated genes between groups a and $\mathrm{b}$. f Commonly downregulated genes between groups $\mathrm{c}$ and $\mathrm{d}$.

B. superbus, which inhabited only habitats at an approximate elevation of $5000 \mathrm{~m}$, in contrast to other high-altitude bumblebee species (Figures 1 and $7 \mathrm{~b}, \mathrm{c})$. The bumblebee Rac1 and AAC2 proteins retain high sequence homology with those of other bees, ants and silkworms, even with those in humans and mice (Figs. S3 and S4). Moreover, based on homology modeling, the bumblebee AAC2 protein shares a conserved structure with known human and bovine AAC2 structures (Figure S5A). The binding $\alpha$ helixes containing $\alpha 2, \alpha 6$, and $\alpha 11$ for the ADP molecule are the same in both insects and humans (Figure S5A and S5B). 
a

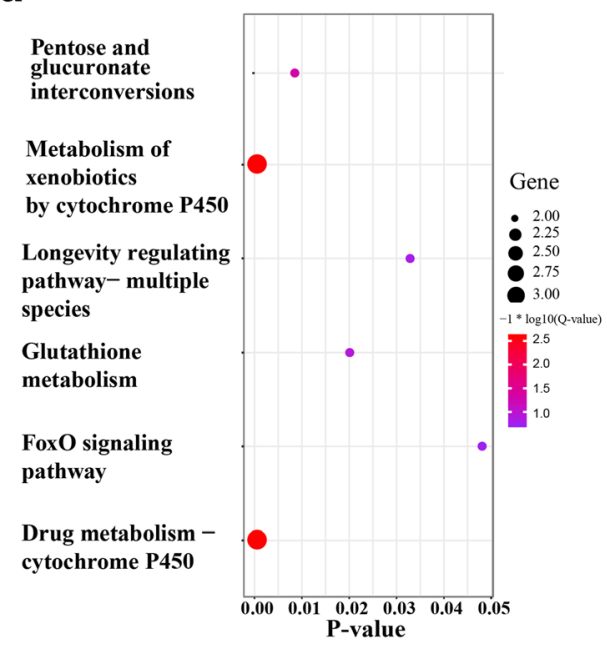

b

Starch and sucrose
metabolism
Pentose phosphate
pathway
Microbial metabolism
in diverse environments
Metabolic pathways
Glycolysis/
Gluconeogenesis
Galactose metabolism
Fructose and mannose
metabolism

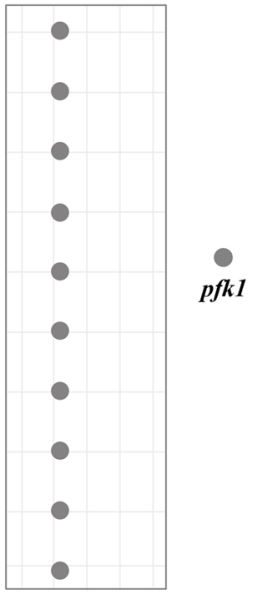

Figure 5. KEGG enrichment analysis of the commonly downregulated genes in the six high-altitude bumblebee species compared to those from the two low-altitude bumblebee species and associated KEGG analysis of the upregulated $p f k 1$ gene. a KEGG enrichment analysis of commonly downregulated genes using the KOBAS 3.0 server (http://http://kobas.cbi.pku.edu.cn/) and the honeybee Apis mellifera database. b KEGG identification of the pfk1 gene based on the honeybee A. mellifera database with the STRING server (https://string-db.org/).

\section{DISCUSSION}

China possesses the greatest bumblebee species diversity in the world, and some species inhabit only the Tibet Plateau (An et al. 2014). Similar to other organisms, these high-altitude bumblebee species have adapted to their severe environment, and this adaptation essentially relies on genome, transcript, and protein regulation. Based on the differentially expressed genes identified in each pairwise comparison of the head and thorax transcriptomes between six high-altitude bumblebee species and two low-altitude species, common differentially expressed genes in all the sequenced bumblebee species were found (Figures 2 and 3). The 141 downregulated genes were significantly involved in the metabolism of xenobiotics by cytochrome P450 and drug metabolism (cytochrome P450), which suggest that toxicity stress was low in the high-altitude bumblebees. The P450 enzyme found in insect tissues performs the synthesis and degradation of ecdysteroids and juvenile hormones and the metabolism of foreign chemicals of natural or synthetic origin (Feyereisen 1999). This phenomenon is consistent with the environment in the Tibet
Plateau, which is more natural than that in the agricultural region of North China, where more disturbances occur due to human activity.

Circulating energy substrates such as trehalose, lipids, and proline ensure a high energy demand during the flight of insects and are replenished by the fat body (Beenakkers et al. 1984). The fat body exists only in insects and is distributed throughout the insect body, facilitating close contact between the hemolymph and fat body cells (Arrese and Soulages 2010). As the circulating sugar in the hemolymph, trehalose at a high concentration in the hemolymph is vital for flight in many insects. Trehalose is synthesized from glucose phosphates and UTP, and trehalose must be reconverted into glucose to be used in cell metabolism (Becker et al. 1996). Bees are an unusual group of insects that exclusively utilize carbohydrates as the main fuel to power flight, and proline plays only an anaplerotic role (Suarez et al. 2005). The three commonly upregulated genes $P f k 1$, $A A C 2$, and $r a c 1$ are closely involved in glycolysis, ATP/ADP transport and glucose transport, which are all crucial processes in the carbohydrate metabolism of bumblebees. The sugars in nectar are mainly sucrose, fructose, and glucose, which 


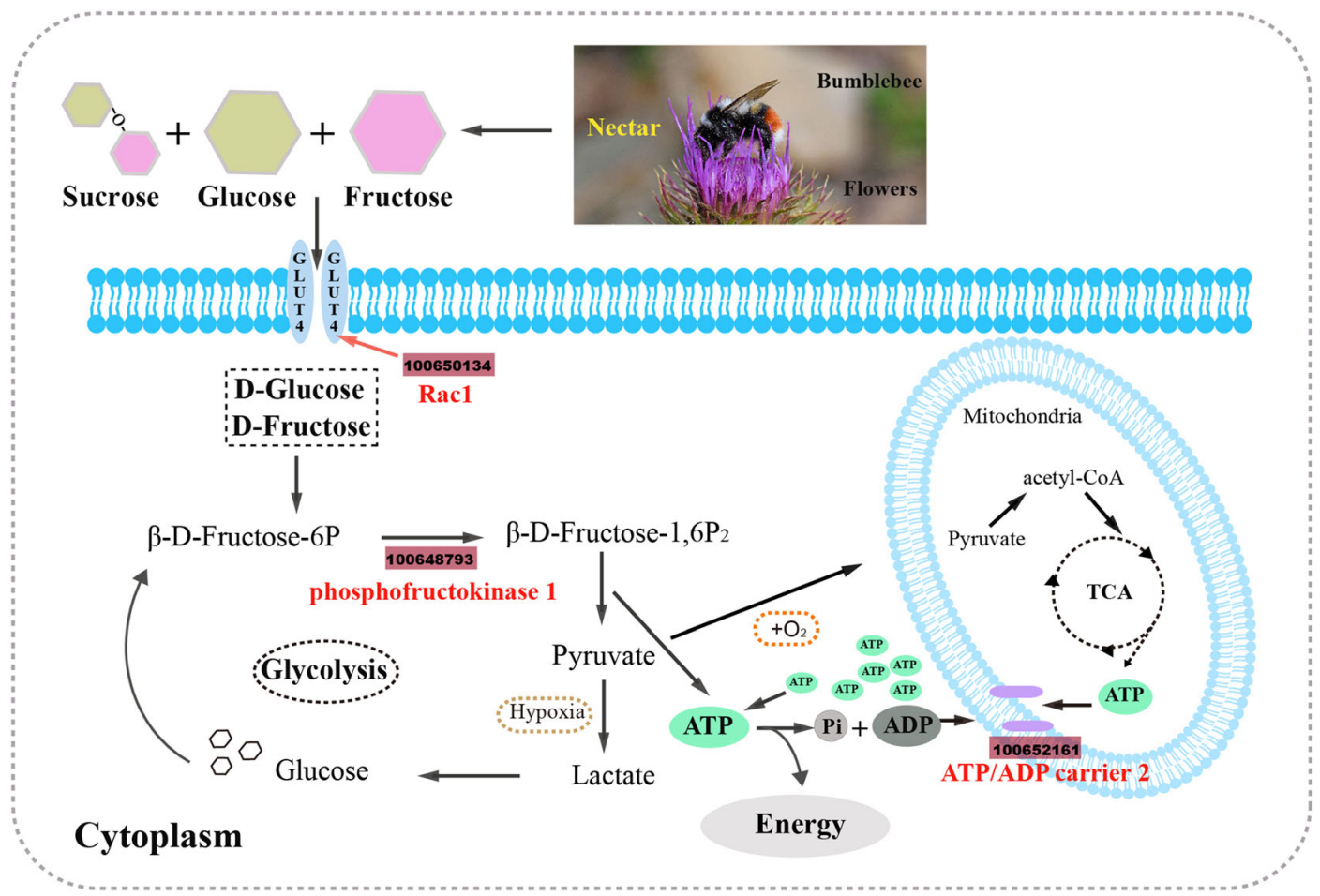

Figure 6. Schematic diagram of the pathways and genes involved in energy regulation in high-altitude Tibetan bumblebees. In the Tibet Plateau environment, the upregulated Racl gene indirectly facilitates the transport of glucose and fructose, which is generally consistent with the route by which food arrives in the cytoplasm and enters glycolysis in bumblebees. The upregulation of the $p f k 1$ gene greatly increases the speed of the committed step of glycolysis, which transforms $\beta$-D-fructose-6P to $\beta$-D-fructose- $1.6 \mathrm{P}_{2}$, which in turn is metabolized to pyruvate and produces ATP molecules. Under a hypoxic environment, the TCA cycle in mitochondria is restricted, and pyruvate is metabolized to lactate, which is transformed to glucose and undergoes glycolysis. In addition, the upregulation of ATP/ADP carrier 2 promotes the exchange of ATP and ADP, indirectly accelerating glycolysis. The names of the commonly upregulated genes are colored red, and their gene IDs are shown in dark-red rectangles. GLUT4: glucose transporter type 4, TCA: the citric acid cycle. The black arrow indicates normal and enhanced metabolic pathways; the red arrow indicates that Rac1 promotes the activity of GLUT4; and the gray arrow indicates the exchange of ATP and ADP. The diagram of glycolysis and the TCA cycle was based on an existing schematic diagram (Berg et al. 2002).

can be directly utilized for glycolysis to produce ATP for energy consumption and can be utilized for the synthesis of trehalose in the fat body. During bumblebee flight, trehalose is released into the hemolymph and reconverted to glucose, which enters glycolysis and produces ATP to support the high energy requirements of flight. The Pfk1 enzyme is the first committed step of glycolysis and plays a critical role in the transfer of $\mathrm{D}$-glucose and $\mathrm{D}$-fructose to $\beta$-D-fructose$1,6 \mathrm{P}_{2}$, the upregulation of which directly facilitates glycolysis to increase ATP production. The bumblebee Pfk1 protein retains highly conserved binding residues for ATP, ADP, ACP, F6P, and F6BP, similar to those in the human Pfk1 found in skeletal muscle; therefore, common upregulation of the $P f k 1$ gene accelerates aerobic and anaerobic glycolysis to produce energy to sustain the flight of high-altitude bumblebees in cold temperatures. Moreover, based on the known bumblebee genomes, EPAS1 orthologs that encode one subunit of hypoxia-inducible factor (HIF) to regulate hypoxic adaptation in vertebrates were absent in bumblebees (Sadd et al. 2015). The absence of the 
a

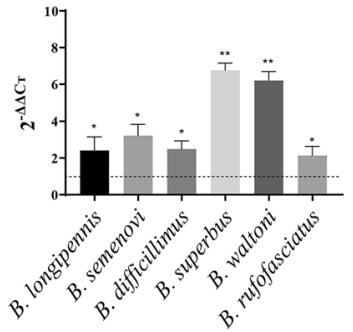

Pfk1

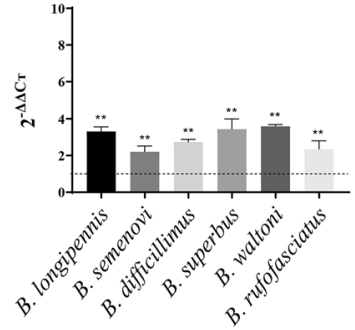

Rac1

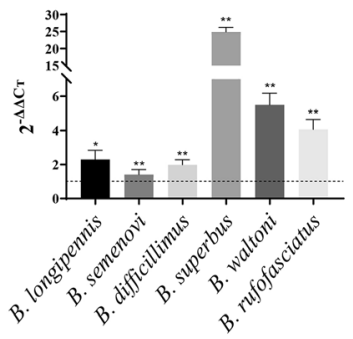

ACC2

b
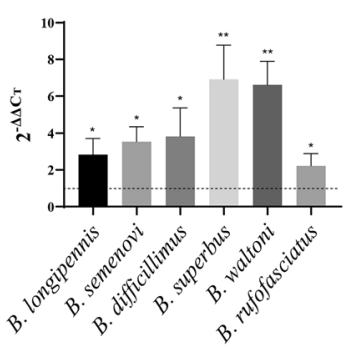

Pfkl

C
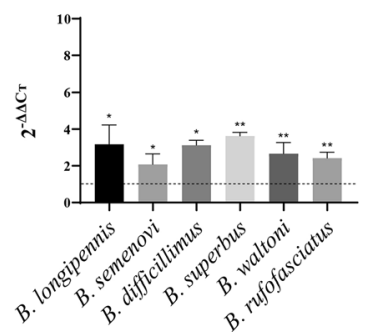

Pfkl

d
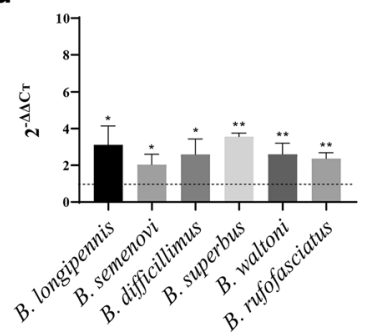

Pfkl

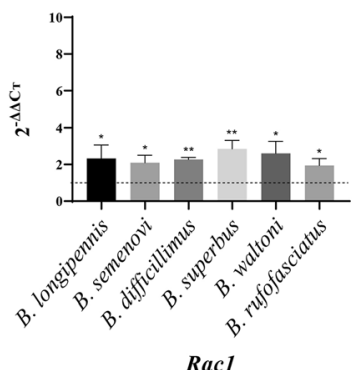

Rac1
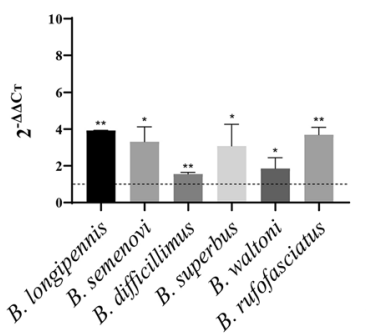

Rac1

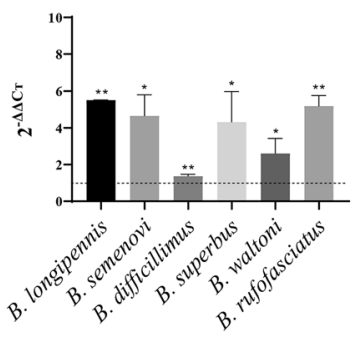

Rac1
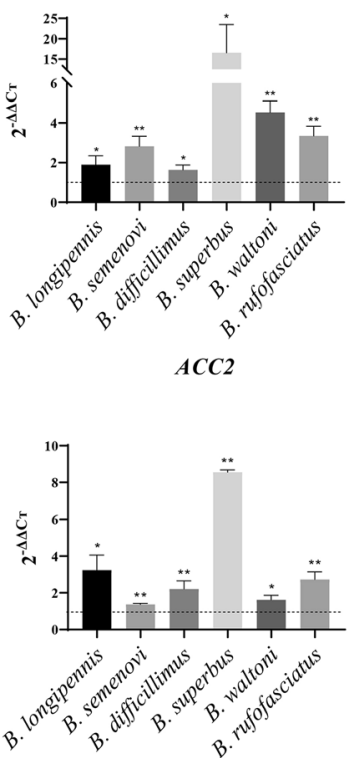

$A A C 2$

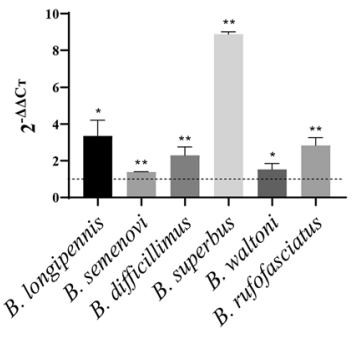

AAC2

Figure 7. Real-time qPCR results of the three commonly upregulated genes, $P f k 1$, Racl and $A A C 2$, in six highaltitude Tibetan bumblebee species. a Based on the a-actin reference gene, the real-time qPCR results of Pfk1, $R a c 1$, and $A A C 2$ of the six high-altitude Tibetan bumblebee species in comparison with $B$. patagiatus are shown. b Based on the a-actin reference gene, the real-time qPCR results of $P f k 1, R a c 1$, and $A A C 2$ of the six high-altitude Tibetan bumblebee species in comparison with $B$. ignitus are shown. c Based on the arginine kinase reference gene, the real-time qPCR results of $P f k 1, R a c 1$, and $A A C 2$ of the six high-altitude Tibetan bumblebee species in comparison with $B$. patagiatus are shown. d Based on the arginine kinase reference gene, the real-time qPCR results of $P f k 1$, Rac1, and $A A C 2$ of the six high-altitude Tibetan bumblebee species in comparison with B. ignitus are shown. The $\mathrm{X}$-axis represents the six high-altitude bumblebee species, the $\mathrm{Y}$-axis represents the values of $2^{-\Delta \Delta \mathrm{Ct}}$, $\rightarrow$ represents $p$ value $<0.05$ and $* *$ represents $p$ value $<0.01$. 
EPAS1 orthologs also suggested that hypoxia might not be the greatest challenge to highaltitude bumblebee survival and flight. Moreover, high-altitude Tibetan bumblebees are capable of enhancing the associated pathways of aerobic metabolism based on the efficient oxygen exchange system in insects (Harrison and Roberts 2000; Dillon et al. 2006). The upregulation of $P f k 1$ could guarantee normal glycogen intake and glycolysis, which produce energy to warm the body at cold temperatures and adapt to hypoxic surroundings. Moreover, the upregulation of $P f k l$ alone cannot guarantee glycolytic flux, which can increase if the glucose-6-phosphate supply is also increased. Therefore, GLUT4 recruitment and the stimulation of glycogen breakdown are intrinsic parts of the multisite concerted mechanism by which glycolysis is stimulated. Racl regulates the translocation of glucose, transporting GLUT4 vesicles from intracellular compartments to the plasma membrane and is also necessary for glucose uptake in skeletal muscles activated by exercise (Ueda et al. 2010; Sylow et al. 2013; Sylow et al. 2014; Sylow et al. 2016). Racl is expressed in significant amounts in adipose tissue and skeletal muscles in mammals. Bumblebee Rac1 possesses a highly conserved structure compared to human Rac1 and contains the same functional domain. Hence, upregulated Rac1 could enhance GLU4 recruitment, thus assisting Pfk1 in vastly accelerating glycolysis to produce energy for the flight and survival of high-altitude bumblebees. However, AAC2, an ADP/ATP carrier, performs the vital role of transporting ADP into the mitochondrial matrix and ATP out of the mitochondrion to maintain high cytosolic ATP concentrations for reactions requiring energy (Ruprecht et al. 2019). Upregulation of bumblebee $A A C 2$ facilitates the exchange of ATP and ADP, which promotes glycolysis.

In conclusion, based on the two low-altitude bumblebee species that served as controls, conjoint analysis of the transcriptomes of eight bumblebee species proved that the Pfkl gene is a significant factor regulating the energy demand of bumblebees on the high-altitude Tibet Plateau against cold temperatures and hypoxic surroundings. Furthermore, the Racl and $A A C 2$ genes also play an important role in energy production by enhancing ATP/ADP exchange and glucose uptake. However, because of the low mapping ratios of the sequenced high-altitude bumblebees (except for B. longipennis ), common differentially expressed genes involved in oxidative phosphorylation and the TCA cycle remained unidentified in all the high-altitude Tibetan bumblebees. These low mapping ratios to the reference genome and the potentially different gene sizes between the different bumblebee species reduced the number of identified differentially expressed genes involved in the regulation of high-altitude adaptation in bumblebees. More genomes of highaltitude bumblebee species from different subgenera need to be resolved to thoroughly understand the regulatory genes involved in highaltitude adaptation. Moreover, the adaptation mechanism of bumblebees on the Tibet Plateau needs to be comprehensively explained by multiple studies involving genome evolution and protein function.

\section{ACKNOWLEDGMENTS}

We are very thankful to Jiaxing Huang and Paul Williams for their help in identifying the bumblebees of this study and to Jiaxing Huang, Yaning Zhang, and Shiwen Zhang for their help in field collecting of the samples.

Data accessibility The transcriptomic data have been deposited to NCBI SRA database. And the accession number as follows:

Bombus longipennis : SRR10811679

Bombus difficillimus : SRR10812190

Bombus rufofasciatus : SRR10812319

Bombus semenovi: SRR10820296

Bombus superbus: SRR10821716

Bombus waltoni : SRR10821769

Bombus ignitus : SRR10821775

Bombus patagiatus : SRR10821878

\section{AUTHOR CONTRIBUTIONS}

JA conceived and designed this research; YL and JA collected the samples; YL and HJ performed the experiments and processed the data and analyzed the data; YL wrote the manuscript with contributions from $\mathrm{MN}$; all authors reviewed and approved the final manuscript. 


\section{FUNDING INFORMATION}

This work was supported by the Natural Science Foundation of China (31672500), the Agricultural Science and Technology Innovation Program (CAAS-ASTIP-2015-IAR), and the Special Program for Basic Resources of Science and Technology (2018FY100404).

\section{COMPLIANCE WITH ETHICAL STANDARDS}

Conflict of interest The authors declare that they have no conflict of interest.

Une analyse comparative du transcriptome révèle des gènes régulateurs impliqués dans la tolérance au froid et l'adaptation hypoxique des bourdons tibétains de haute altitude.

Pollinisateurs / bourdons / Plateau du Tibet / transcriptome / métabolisme énergétique / pfk1.

Eine vergleichende Transkriptomanalyse zeigt Gene auf, die eine Rolle spielen können in der Kältetoleranz und Anpassung an den Sauerstoffmangel bei Hochlandhummeln in Tibet.

Bestäuber / Hummeln / Tibet-Plateau / Transkriptom / Energiemetabolismus / pfk1.

\section{REFERENCES}

Altshuler, D.L., Dudley, R. (2006) The physiology and biomechanics of avian flight at high altitude. Integr Comp Biol 46 (1), 62-71.

An, J., Huang, J., Shao, Y., Zhang, S., Wang, B., Liu, X., $\mathrm{Wu}$, J., Williams, P.H. (2014) The bumblebees of North China (Apidae, Bombus Latreille). Zootaxa 3830.

Archana, M.N., Archana, N.P. (2016) Glucose transporters: physiological and pathological roles. Biophys Rev 8 (1), 5-9.

Arrese, E.L., Soulages, J.L. (2010) Insect fat body: energy, metabolism, and regulation. Annu Rev Entomol 55, 207-225.

Beall, C.M., Cavalleri, G.L., Deng, L., Elston, R.C., Gao, Y., et al. (2010) Natural selection on EPAS1 (HIF2alpha) associated with low hemoglobin concentration in Tibetan highlanders. Proc Natl Acad Sci U S A 107 (25), 11459-11464.

Becker, A., Schlöder, P., Steele, J.E., Wegener, G. (1996) The regulation of trehalose metabolism in insects. Experientia 52, 433-439.

Beenakkers, A.M., VanderHorst, D.J., Marrewijk, W.J.A. (1984) Insect flight muscle metabolism. Insect Biochem 14 (3), 243-260.

Berg, J.M., Tymoczko, J.L., Stryer, L. (2002) Glycolysis and Gluconeogenesis. Biochemistry, 5rd edn. W H Freeman, New York, Chapter 16.

Bigham, A., Bauchet, M., Pinto, D., Mao, X., Akey, J.M., et al. (2010) Identifying signatures of natural selection in Tibetan and Andean populations using dense genome scan data. PLoS Genet 6 (9), e1001116.

Blatt, J., Roces, F. (2001) Haemolymph sugar levels in foraging honeybees (Apis mellifera carnica): dependence on metabolic rate and in vivo measurement of maximal rates of trehalose synthesis. J Exp Biol 204 (Pt15), 2709-2716.

Brahimi-Horn, M.C., Chiche, J., Pouyssegur, J. (2007) Hypoxia signalling controls metabolic demand. Curr Opin Cell Biol 19 (2), 223-229.

Dillon, M.E., Dudley, R. (2014) Surpassing Mt. Everest: extreme flight performance of alpine bumble-bees. Biol Lett 10 (2), 20130922.

Dillon, M.E., Frazier, M.R. (2006) Drosophila melanogaster locomotion in cold thin air. J Exp Biol 209 (Pt2), 364-371.

Dillon, M.E., Frazier, M.R., Dudley, R. (2006) Into thin air: Physiology and evolution of alpine insects. Integr Comp Biol 46 (1), 49-61.

Feyereisen, R. (1999) Insect P450 enzymes. Annu Rev Entomol 44, 507-533.

Galen, S.C., Natarajan, C., Moriyama, H., Weber, R.E., Fago, A., Benham, P.M., Chavez, A.N., Cheviron, Z.A., Storz, J.F., Witt, C.C. (2015) Contribution of a mutational hot spot to hemoglobin adaptation in highaltitude Andean house wrens. Proc Natl Acad Sci U S A 112 (45), 13958-13963.

Gatenby, R.A., Gillies, R.J. (2004) Why do cancers have high aerobic glycolysis? Nat Rev Cancer 4 (11), 891-899.

Ge, R.L., Cai, Q., Shen, Y.Y., San, A., Ma, L., et al. (2013) Draft genome sequence of the Tibetan antelope. Nat Commun: https://doi.org/10.1038/ncomms2860.

Ghabrial, A., Luschnig, S., Metzstein, M.M., Krasnow, M.A. (2003) Branching morphogenesis of the Drosophila tracheal system. Annu Rev Cell Dev Biol 19, 623-647.

Gmeinbauer, R., Crailsheim, K. (1993) Glucose utilization during flight of honeybee (Apis mellifera) workers, drones and queens. J Insect Physiol 39 (11), 959-967.

Gou, X., Wang, Z., Li, N., Qiu, F., Xu, Z., et al. (2014) Whole-genome sequencing of six dog breeds from continuous altitudes reveals adaptation to highaltitude hypoxia. Genome Res 24 (8), 1308-1315.

Harrison, J.F., Roberts, S.P. (2000) Flight respiration and energetics. Annu Rev Physiol 62, 179-205. 
Hodkinson, I.D. (2005) Terrestrial insects along elevation gradients: species and community responses to altitude. Biol Rev Camb Philos Soc 80 (3), 489-513.

Huang, J., An, J. (2018) Species diversity, pollination application and strategy for conservation of the bumblebees of China. Biodiversity Science 26 (5), 486-497.

Huey, R.B., Kingsolver, J.G. (1989) Evolution of thermal sensitivity of ectotherm performance. Trends Ecol Evol 4 (5), 131-135.

Josephson, R.K. (1981) Temperature and the mechanical performance of insect muscle. In: Insect Thermoregula tion (B. Heinrich, ed.), John Wiley, New York, pp. 1944.

Klip, A., Sun, Y., Chiu, T.T., Foley, K.P. (2014) Signal transduction meets vesicle traffic: the software and hardware of GLUT4 translocation. Am J Physiol Cell Physiol 306 (10), C879-886.

Liu, X., Zhang, Y., Li, Y., Pan, J., Wang, D., et al. (2019) EPAS1 Gain-of-Function Mutation Contributes to High-Altitude Adaptation in Tibetan Horses. Mol Biol Evol 36 (11), 2591-2603.

Mani, M.S. (1968) The High Altitude Environment. In: Ecology and Biogeography of High Altitude Insects. Series Entomologica, vol 4. Springer, Dordrecht.

Miyazawa, H., Yamaguchi, Y., Sugiura, Y., Honda, K., Kondo, K., Matsuda, F., Yamamoto, T., Suematsu, M., Miura, M. (2017) Rewiring of embryonic glucose metabolism via suppression of PFK-1 and aldolase during mouse chorioallantoic branching. Development 144 (1), 63-73.

Mor, I., Cheung, E.C., Vousden, K.H. (2011) Control of glycolysis through regulation of PFK1: old friends and recent additions. Cold Spring Harb Symp Quant Biol 76, 211-216.

Natarajan, C., Projecto-Garcia, J., Moriyama, H., Weber, R.E., Munoz-Fuentes, V., et al. (2015) Convergent Evolution of Hemoglobin Function in High-Altitude Andean Waterfowl Involves Limited Parallelism at the Molecular Sequence Level. PLoS Genet 11 (12), e1005681.

Natarajan, C., Hoffmann, F.G., Weber, R.E., Fago, A., Witt, C.C., Storz, J.F. (2016) Predictable convergence in hemoglobin function has unpredictable molecular underpinnings. Science 354 (6310), 336-339.

Nunes, R.D., Romeiro, N.C., De Carvalho, H.T., Moreira, J.R., Sola-Penna, M., Silva-Neto, M.A., Braz, G.R. (2016) Unique PFK regulatory property from some mosquito vectors of disease, and from Drosophila melanogaster. Parasit Vectors, DOI: https://doi. org/10.1186/s13071-016-1391-y.

Peng, Y., Yang, Z., Zhang, H., Cui, C., Qi, X., et al. (2011) Genetic variations in Tibetan populations and highaltitude adaptation at the Himalayas. Mol Biol Evol 28 (2), 1075-1081.

Peng, Y., Cui, C., He, Y., Ouzhuluobu, Zhang, H., et al. (2017) Down-Regulation of EPAS1 Transcription and Genetic Adaptation of Tibetans to High-Altitude Hypoxia. Mol Biol Evol 34 (4), 818-830.
Projecto-Garcia, J., Natarajan, C., Moriyama, H., Weber, R.E., Fago, A., Cheviron, Z.A., Dudley, R., McGuire, J.A., Witt, C.C., Storz, J.F. (2013) Repeated elevational transitions in hemoglobin function during the evolution of Andean hummingbirds. Proc Natl Acad Sci U S A 110 (51), 20669-20674.

Qiu, Q., Zhang, G., Ma, T., Qian, W., Wang, J., et al. (2012) The yak genome and adaptation to life at high altitude. Nat Genet 44 (8), 946-949.

Roy, R., Schmitt, A.J., Thomas, J.B., Carter, C.J. (2017) Review: Nectar biology: From molecules to ecosystems. Plant Sci 262, 148-164.

Royden, L.H., Burchfiel, B.C., van der Hilst, R.D. (2008) The geological evolution of the Tibetan Plateau. Science 321 (5892), 1054-1058.

Ruprecht, J.J., King, M.S., Zogg, T., Aleksandrova, A.A., Pardon, E., Crichton, P.G., Steyaert, J., Kunji, E.R.S. (2019) The Molecular Mechanism of Transport by the Mitochondrial ADP/ATP Carrier. Cell 176 (3), 435447 e415.

Sacktor, B. (1976) Biochemical adaptations for flight in the insect. Biochem Soc Symp, 41, 111-131.

Sacktor, B., Wormser-Shavit, E. (1966) Regulation of metabolism in working muscle in vivo. I. Concentrations of some glycolytic, tricarboxylic acid cycle, and amino acid intermediates in insect flight muscle during flight. J Biol Chem 241 (3), 624-631.

Sadd, B.M., Barribeau, S.M., Bloch, G., de Graaf, D.C., Dearden, P., et al. (2015) The genomes of two key bumblebee species with primitive eusocial organization. Genome Biol, DOI: https://doi.org/10.1186 /s13059-015-0623-3.

Scaraffia, P.Y., Gerez de Burgos, N.M. (2000) Effects of temperature and $\mathrm{pH}$ on hexokinase from the flight muscles of Dipetalogaster maximus (Hemiptera: Reduviidae). J Med Entomol 37 (5), 689-694.

Sharma, B. (2011) Kinetic Characterisation of Phosphofructokinase Purified from Setaria cervi: A Bovine Filarial Parasite. Enzyme Res, DOI: https://doi. org/10.4061/2011/939472.

Simonson, T.S., Yang, Y., Huff, C.D., Yun, H., Qin, G., et al. (2010) Genetic evidence for high-altitude adaptation in Tibet. Science 329 (5987), 72-75.

Suarez, R.K. (2000) Energy metabolism during insect flight: biochemical design and physiological performance. Physiol Biochem Zool. 73 (6), 765-771.

Suarez, R.K., Darveau, C.A., Welch, K.C., Jr., O'Brien, D.M., Roubik, D.W., Hochachka, P.W. (2005) Energy metabolism in orchid bee flight muscles: carbohydrate fuels all. J Exp Biol 208 (Pt18), 3573-3579.

Sylow, L., Jensen, T.E., Kleinert, M., Mouatt, J.R., Maarbjerg, S.J., Jeppesen, J., Prats, C., Chiu, T.T., Boguslavsky, S., Klip, A. (2013) Rac1 is a novel regulator of contraction-stimulated glucose uptake in skeletal muscle. Diabetes 62 (4), 1139-1151.

Sylow, L., Kleinert, M., Pehmoller, C., Prats, C., Chiu, T.T., Klip, A., Richter, E.A., Jensen, T.E. (2014) Akt and Rac1 signaling are jointly required for insulinstimulated glucose uptake in skeletal muscle and 
downregulated in insulin resistance. Cell Signal 26 (2), 323-331.

Sylow, L., Nielsen, I.L., Kleinert, M., Møller, L.L., Ploug, T., Schjerling, P., Bilan, P.J., Klip, A., Jensen, T.E., Richter, E.A. (2016) Rac1 governs exercise-stimulated glucose uptake in skeletal muscle through regulation of GLUT4 translocation in mice. J Physiol 594 (17), 4997-5008.

Thaís, D.B., Thiago, G.P.A., Walter, R.T. (2010) Transporters involved in glucose and water absorption in the Dysdercus peruvianus (Hemiptera: Pyrrhocoridae) anterior midgut. Comp Biochem Physiol B Biochem Mol Biol 157 (1), 1-9.

Ueda, S., Kitazawa, S., Ishida, K., Nishikawa, Y., Matsui, M., et al. (2010) Crucial role of the small GTPase Rac1 in insulin-stimulated translocation of glucose transporter 4 to the mouse skeletal muscle sarcolemma. FASEB J 24 (7), 2254-2261.

Wigglesworth, V.B. (1972) The Principles of Insect Physiology. 8th Edition. Chapman and Hall, London, pp. 663-699.

Williams, P.H., Ito, M., Matsumura, T., Kudo, I. (2010) The bumblebees of the Nepal Himalaya (Hymenoptera: Apidae). Insecta Matsumurana 66, 115-151.

Williams, P.H., Huang, J., Rasmont, P., An, J. (2016) Early-diverging bumblebees from across the roof of the world: the high-mountain subgenus $<\mathrm{i}>$ Mendacibombus $</ \mathrm{i}>$ revised from species' gene coalescents and morphology (Hymenoptera, Apidae). Zootaxa 4204

Xie, C., Mao, X., Huang, J., Ding, Y., Wu, J., Dong, S., Kong, L., Gao, G., Li, C.Y., Wei, L. (2011) KOBAS 2.0: a web server for annotation and identification of enriched pathways and diseases. Nucleic Acids Res 39, W316-322.

Xu, S., Li, S., Yang, Y., Tan, J., Lou, H., et al. (2011) A genome-wide search for signals of high-altitude adaptation in Tibetans. Mol Biol Evol 28 (2), 1003-1011.

Yi, X., Liang, Y., Huerta-Sanchez, E., Jin, X., Cuo, Z.X., et al. (2010) Sequencing of 50 human exomes reveals adaptation to high altitude. Science 329 (5987), 75-78.

Zhang, W., Fan, Z., Han, E., Hou, R., Zhang, L., et al. (2014) Hypoxia adaptations in the grey wolf (Canis lupus chanco) from Qinghai-Tibet Plateau. PLoS Genet 10 (7), e1004466.

Zhu, X., Guan, Y., Signore, A.V., Natarajan, C., DuBay, S.G., et al. (2018) Divergent and parallel routes of biochemical adaptation in high-altitude passerine birds from the Qinghai-Tibet Plateau. Proc Natl Acad Sci U S A 115 (8), 1865-1870.

Publisher's note Springer Nature remains neutral with regard to jurisdictional claims in published maps and institutional affiliations. 